\title{
Low-Cost Flow Visualization for a Supersonic Ejector
}

\author{
George W. Olden ${ }^{*}$, David M. Lineberry ${ }^{\dagger}$, Christopher A. B. Linn ${ }^{\ddagger}$ \\ Dr. Brian D. Landrum ${ }^{8}$, and Dr. Clark W. Hawk \\ University of Alabama in Huntsville, Propulsion Research Center, Huntsville, AL 35899
}

\begin{abstract}
Shadowgraph techniques were applied to the cold flow ejector facility at the Propulsion Research Center at the University of Alabama in Huntsville. The setup for the experiments was relatively simple and was accomplished at very little cost. Series of shadowgraph images were taken of both dual nozzle and single nozzle strut based ejectors operating over a range of chamber pressures. The density gradient patterns in the shadowgraphs were compared to pressure data measured along the top and side walls of the mixing duct. The shadowgraph images showed the presence of barrel shocks emanating from the nozzles which at low pressures terminated in Mach disks and at higher pressures extended beyond the barrel shape and reflected off the walls of the duct. Based on pressure data from previous testing, reflected shocks were expected on the walls of the duct. The shadowgraph images confirmed the locations of these reflected shocks on the top wall of the duct. The shadowgraph images also showed the structure change which correlated to a change in pitch of the ejector noise, and corresponded to a change in trend of the duct wall pressure ratio distributions. The images produced from the setup provided insight into the complex flow behavior inside the ejector duct. In addition, the techniques were a valuable tool as an educational device for students.
\end{abstract}

\section{Introduction}

Combined Cycle Propulsion (CCP) technology shows promise for next generation launch vehicles. ${ }^{2}$ Since a combined cycle engine incorporates several modes of engine operation into the same flow path, the optimum performance mode can be utilized in each flight regime. A typical Rocket Based Combined Cycle (RBCC) engine would operate in a rocket or ducted rocket mode for takeoff and initial acceleration to about Mach 2-3, transition to ramjet mode until Mach 4-6, and then transition to scramjet operation. Above Mach 6-8, scramjet operation is unrealistic, and the engine would operate as a pure rocket to accelerate into orbit.

Many CCP concepts incorporate ejectors in the low speed modes (takeoff and initial acceleration), ${ }^{3,4}$ An ejector uses a high speed primary flow to induce a secondary flow from the surroundings. In a rocket based application, the secondary flow is atmospheric air, and thus contains oxygen which can be combusted with fuel rich exhaust. The products from this combustion can be accelerated through a nozzle to provide thrust.

The Propulsion Research Center (PRC) at the University of Alabama in Huntsville (UAH) has an ongoing program to study fluid mechanics in a cold flow ejector. The facility is based on the StrutJet concept ${ }^{3}$ and utilizes air as both the primary and the secondary flows. Previous research at the PRC have presented quantative information on the fluid mechanics in the PRC facility. ${ }^{5-8}$ Results from these tests have provided information on the pressure distribution throughout the duct, mass flow rates of the primary and secondary flow streams, choking phenomena in the duct, and Mach numbers of the flow. Up to this point, little work has been done to provide flow visualization within the duct that could be correlated to the measurements made in the tests. Some of the initial work at the PRC was focused on mixing between the exhaust of a dual nozzle strut and a turbine slit located between

\footnotetext{
- Research Technician,, Mechanical and Aerospace Engineering Department, S225 Technology Hall, Huntsville AL, 35899, Student Member

${ }^{+}$Graduate Research Assistant, Mechanical and Aerospace Engineering Department, S225 Technology Hall,

Huntsville AL, 35899, Student Member

${ }^{\ddagger}$ Graduate Research Assistant, Mechanical and Aerospace Engineering Department, S225 Technology Hall, Huntsville AL, 35899, Student Member

${ }^{\S}$ Associate Professor, Mechanical and Aerospace Engineering Department, S225 Technology Hall, Huntsville, AL, 35899, AIAA Associate Fellow

• Director, Propulsion Research Center, S225 Technology Hall, Huntsville, AL, 35899, AIAA Fellow
}

This material is declared a work of the U.S. Government and is not subject to copyright protection in the United States. 
the two nozzles. ${ }^{7}$ Planer Induced Laser Fluorescence techniques were used to examine mixing between the nozzle exhaust and the turbine exhaust. However, this technique did not provide much information about the flow structures in the duct. Some shadowgraph images of the dual nozzle strut were made early in the program ${ }^{9}$, however few measurements were made for those tests, and the duct was not instrumented as thoroughly as it is in the present state. No visualizations of the single nozzle strut had been performed prior to this effort.

The use of shadowgraph and Schlieren techniques in inhomogeneous media has been around for centuries. The first published image of a shadowgraph appears in a volume on the physics of fire by Jean Paul Marat which was published in 1780. The image was a drawing of thermal plumes of heated metal objects. ${ }^{1}$ Early Schlieren works date back to Robert Hooke (1635-1703). ${ }^{1}$ Although modern Schlieren techniques require expensive lenses and mirrors to produce good results, shadowgraph techniques can be applied to transparent fluid flow facilities at a relatively low cost. Depending on the flow under investigation, the images produced through shadowgraphy can provide valuable insight to flow behavior as well as add to academic understanding of flow properties. This paper presents the results of shadowgraph imaging performed on the PRC ejector facility. The work was performed with materials that were either on hand, or easily acquired at relatively no cost, and with little setup. The images that were produced were edited with computer software that is fairly standard or can be easily acquired.

\section{Theory}

Reference 1 discusses various shadowgraph and Schlieren techniques and was the primary source of information for the shadowgraph setup used in this project. For ease of construction, the simplest method, known as direct shadowgraphy, was chosen for the ejector facility. Figure 1 shows a diagram of the setup for the direct shadowgraph method. The only requirements for this system are a point light source, the flow region of interest, and a flat screen onto which the shadow is projected. A shadowgraph is formed anytime the media between the light source and the screen is inhomogeneous with respect to density. As light passes through the test region, different densities cause different refractive indexes which cause the light rays from the source to be bent by varying degrees. The refracted rays illuminate the screen at some height, $\Delta \mathrm{h}$, above or below the position where the non refracted ray would have hit. This causes a brighter spot where the light ray hits, and a darker spot where the ray would have hit if the path was not altered. When all of these rays travel along their different paths and arrive at the screen nonuniformly, a shadowgraph is formed.

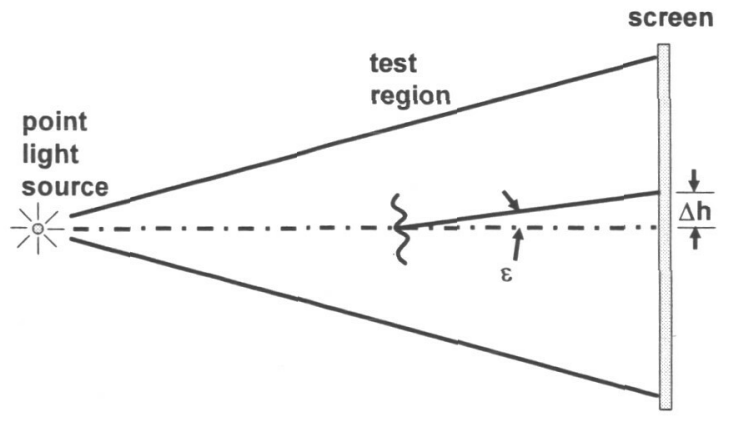

Figure 1. direct shadowgraph method ${ }^{1}$

A true point light source is not even required realizing that the Sun, having an apparent visual angle of 0.5 degrees, can cast shadowgraphs on a sunny day. ${ }^{1}$ An example of this is seen in the shadow cast by gas fumes at a filling station. The spatial relationship between the light source, media to be imaged, and the screen have the greatest effect on the sharpness and sensitivity of the shadowgraph image. ${ }^{1}$ In order to obtain the highest sharpness and sensitivity the light source should have the smallest practical aperture to approximate a point. Also, the distance between the media to be imaged and the screen should be as small as possible when compared to the distance between the light source and the screen. In practical laboratory setups where the main focus of the research is not necessarily the flow visualization, it is not always economical to find a point light source with enough intensity to create useful shadowgraph images. However, as mentioned earlier a true point light source is not required. As the light source becomes larger, the distance between the test region and the light source can be increased to compensate. The goal of moving the light away is minimize the apparent visual angle. Additionally, if the light source is too bright, some of the light can be blocked at the source. For the shadowgraphs presented in this paper, a 
cardboard box was put around the light with a small hole created by a washer taped over a cutout in the box. This method produced very effective shadowgraphs as will be shown later.

\section{Facility Description}

The UAH PRC cold flow ejector facility was originally designed to approximate the flow path of the Aerojet Strutjet concept. ${ }^{3}$ As shown schematically in Fig. 2, the flow path consists of a rectangular cross section duct 4.5 in. wide by 3 in. tall. An elliptically contoured converging inlet is located on the front end of the duct to provide uniform airflow from the laboratory. The mixing duct has 0.75 in. Plexiglas side walls with 0.75 in. aluminum top and bottom walls. A single strut is installed in the duct approximately 25 inches downstream of the inlet. The PRC has been experimenting with two struts, a single nozzle strut and a dual nozzle strut. The struts, shown in Fig. 3, have identical rectangular cross sections 1 inch wide by 7 inches tall. Rocket nozzles are embedded in the struts to provide the primary flow stream for the system. These nozzles have identical geometries with area ratios of approximately 4.66 and transition from a circular throat to a square exit. The dual nozzle strut also has a turbine exhaust slit approximately 0.094 in wide located between the two rocket nozzles. For this series of testing, the slit was not utilized, and was therefore filled.

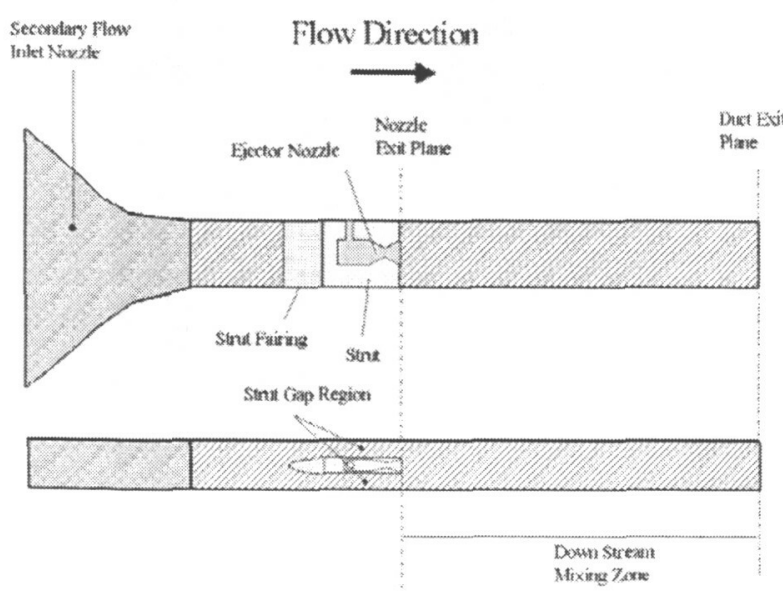

Figure 2. UAH strut ejector flow path (Not to Scale).
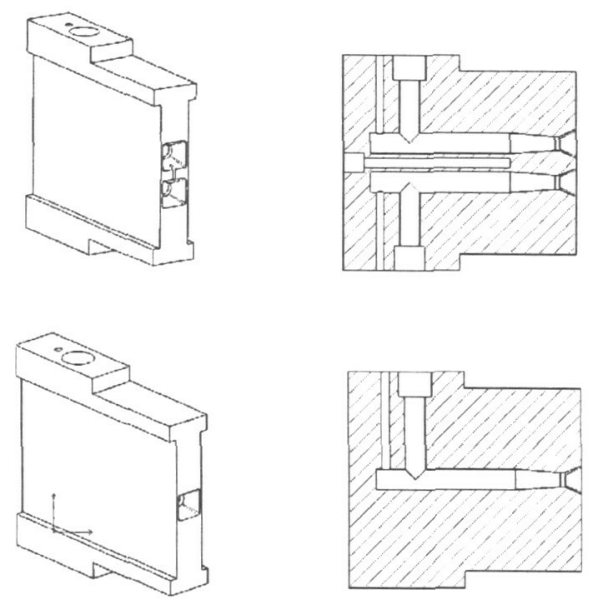

Figure 3. CAD drawings of dual and single nozzle struts.

The PRC ejector facility is shown in Fig. 4. The facility consists of two air tanks with a combined volume of 524 cubic feet, 1.25 in. stainless steel supply tubing, a PID controller to regulate chamber pressure, and the duct. A single strut is installed in the duct for a particular test. An aerodynamic fairing is located on the upstream end of the strut to provide uniform flow in the strut gap region. The duct is instrumented with various pressure taps along the mixing duct and in the strut gap region as well as with pitot-static probes at the upstream fairing and at the exit, and thermocouples in the rocket chamber and at the inlet. Qualitative results from these pressure taps are presented in a parallel paper and will be used for this paper on an as needed basis. ${ }^{10}$ A pressure tap is also located in the chamber of the rocket nozzle to measure the chamber pressure of the rocket. The chamber pressure measurement is connected to a feedback loop of the PID controller to maintain a relatively constant chamber pressure for the experiment.

This work focuses on the region of the duct from the exit plane of the struts to a position approximately 15 inches downstream in the mixing duct. Figure 5 shows the region of interest for the shadowgraph images presented in this paper. In this region, the high pressure primary flow streams are exiting the strut nozzles and the entrained secondary flow is entering the mixing duct around the strut. Strong shock structure forms at the exit of the nozzles, as the primary flow stream tries to match static pressure in the region. These strong shock features make shadowgraphy an appealing technique for flow visualization. 


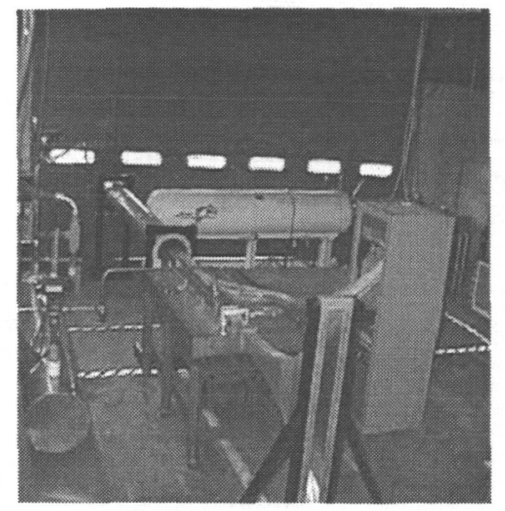

Figure 4. UAH PRC ejector facility.

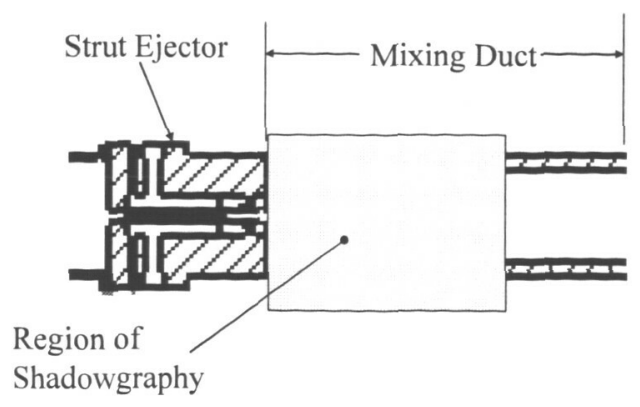

Flow Direction

Figure 5. Region of interest for shadowgraphy.

Figure 6 shows a diagram of the shadowgraph setup used for this study. The light source used was a lamp from a $16 \mathrm{~mm}$ projector. A box was placed in front of the lamp to reduce the light. A washer was mounted over a hole cut in box, and the light from the lamp shone through the hole in the washer. This hole allowed the setup to closer approximate a point light source and reduced washout effects from an overly intense light source. The lamp and box were located approximately $20 \mathrm{ft}$ away from the ejector duct. The screen for this series of experiments consisted of a thin sheet of vellum which was mounted to a cardboard box which had the bottom cut out. The screen was mounted approximately 5 inches away from the duct on the side opposite the light source as seen in Fig. 6 . A digital SLR camera was mounted on a tripod behind the screen and was used to capture the images. For this experiment, the only cost associated was the cost of the vellum. The projector was found in university surplus, but other light sources were tried which produced similar results. The camera was the only other piece of equipment which would have any appreciable cost. Most facilities have digital cameras available, however, a film camera would work equally well, once the lighting and exposure settings were appropriately fixed.

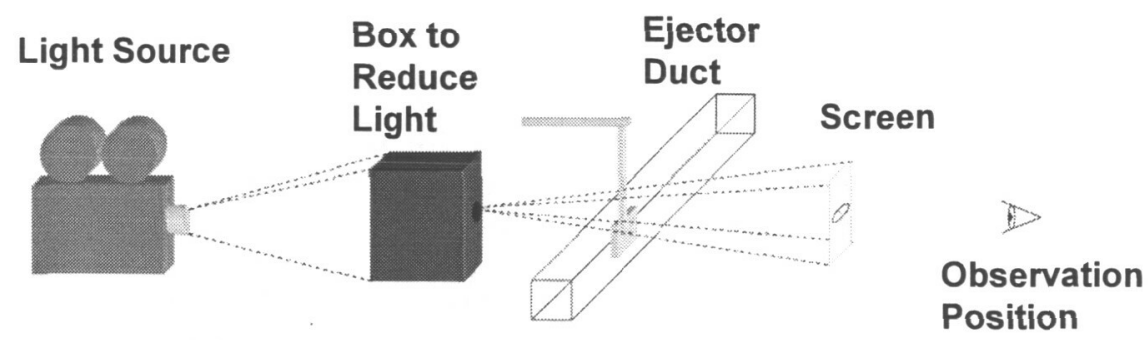

Figure 6. Schematic of shadowgraph setup for ejector facility.

\section{Results}

Shadowgraphy was performed for a series of tests on both the single and dual nozzle strut. For the single nozzle strut, the chamber pressure was varied from $100 \mathrm{psi}$ to $900 \mathrm{psi}$ in $100 \mathrm{psi}$ increments. For the dual nozzle strut, the rocket chamber pressures were varied from 50 psi to 450 psi in 50 psi increments. These chamber pressures provide equivalent mass flow rates i.e. a $50 \mathrm{psi}$ chamber pressure in the dual nozzle has an equivalent mass flow as a 100 psi chamber pressure in the single nozzle strut. It was found that pressure distribution trends in the mixing duct were a function of the primary mass flow rate rather than the nozzle exit pressure. ${ }^{10}$ The shadowgraph images were taken while the pressure was ramped up, and while the tests were at quasi-steady state. The shadowgraphs were compared to the pressure data taken along the top and side wall of the duct.

\section{A. Single Nozzle Results}

Using shadowgraphy on the ejector experiment allowed the structure of primary and secondary flow interactions to be seen. First, shadowgraphs illustrated how the flow in the duct develops and how the shock structure changes 
in response to changes in chamber pressure. Second, the shadowgraphs show how the top and side wall pressure profiles along the duct correlate to the mixing characteristics. Figure 7 shows a series of four shadowgraphs at steady state conditions for four different chamber pressures. These images are representative of ramping up the chamber pressure to a pressure of $700 \mathrm{psi}$. Figure 7a shows a steady state image at a chamber pressure of 200 psi. The shadowgraphs shows barrel shocks emanating from the nozzle and terminating in a Mach disk at a location approximately 0.6 inches downstream of the nozzle exit. Figure $7 \mathrm{~b}$ shows the steady state image at a chamber pressure of $500 \mathrm{psi}$. In this figure, the barrel shocks have stretched and the Mach disk has widened and moved to a position approximately 2.0 inches downstream of the nozzle exit. Figure 7c shows the steady state image at a chamber pressure of $600 \mathrm{psi}$. In this figure, the barrel shocks have stretched even farther. The Mach disk does not appear as sharp in the image and has moved to a position approximately 2.5 inches downstream. The final image, Fig. $7 \mathrm{~d}$, shows the steady state image at a chamber pressure of $700 \mathrm{psi}$. In this image, the barrel shocks have stretched even further, and intersect at a point approximately 4 inches downstream. The Mach disk has completely disappeared at this point. The transition from 600 to $700 \mathrm{psi}$ has several interesting features. This transition is accompanied by a distinct change in pitch of the ejector "roar". The mixing duct pressure distribution plots also show a change in trend. Figure 8 shows plots of the mixing duct wall pressure distribution for chamber pressures of $600 \mathrm{psi}$ and $700 \mathrm{psi}$. The pressure ratio shown on the figure is the ratio of the wall static pressure to the secondary flow stagnation pressure. At low pressures, the pressure distribution shows a low pressure starting at the nozzle then a recovery region to a location approximately 20 inches downstream where the pressure ratio reaches a value of 1 . This is the point in the mixing duct where the primary and secondary flows are considered mixed. As the chamber pressure is increased above this point, the pressure distribution plot shows a low pressure at the nozzle exit followed by a region of decreasing pressure to a value of approximately 0.3 . The pressure ratio remains relatively constant for a length of the duct then begins to recover to the pressure ratio of 1 . This change in trend indicates that the two flows do not mix as well for higher chamber pressures. Pressure traces at the exit of the duct indicate a large pressure gradient across the exit which confirms that there was poor mixing in the duct. As the strut rocket chamber pressure is increased above the transition point, the recovery point moves further downstream. The shadowgraphs at higher chamber pressures did not reveal any new structure. The only changes in the shadowgraphs were additional stretching and widening of the barrel shock. The transition does not always occur at the same pressure. However. it is currently unknown to what extent other environmental factors such as temperature and humidity affect the flow transition.

(a)
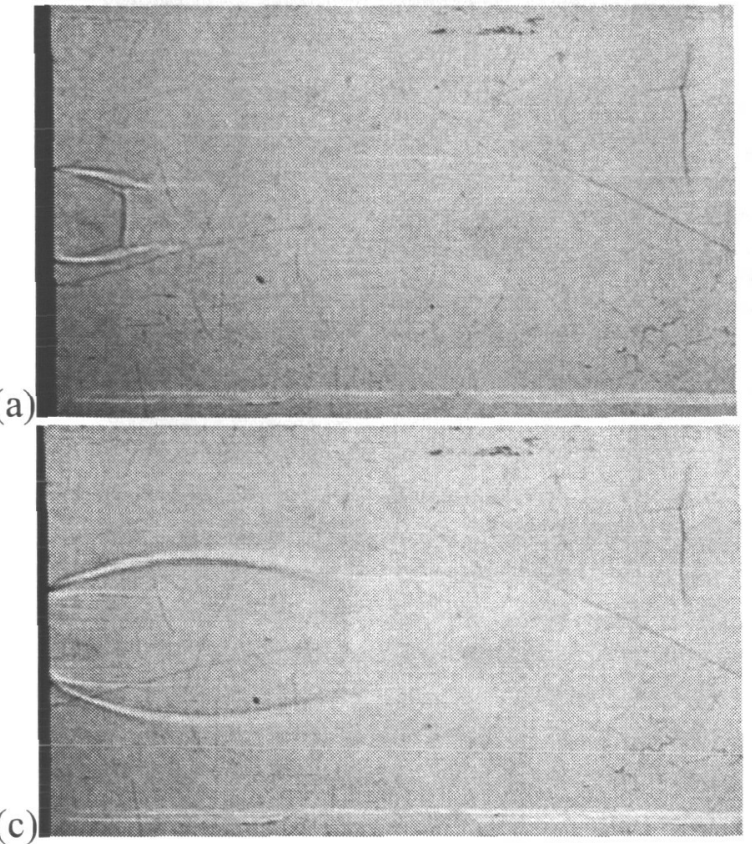

(b)

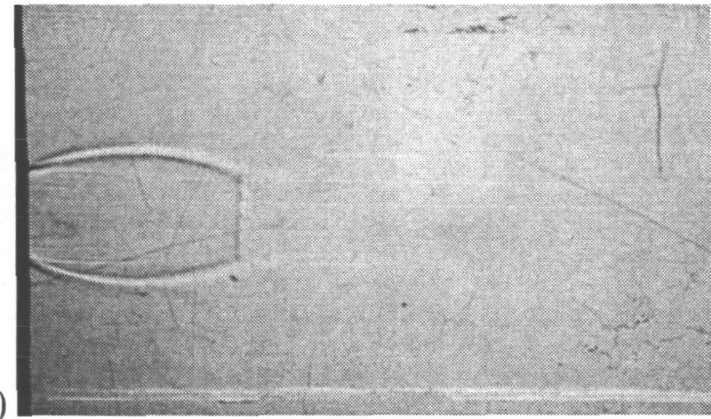

(d)

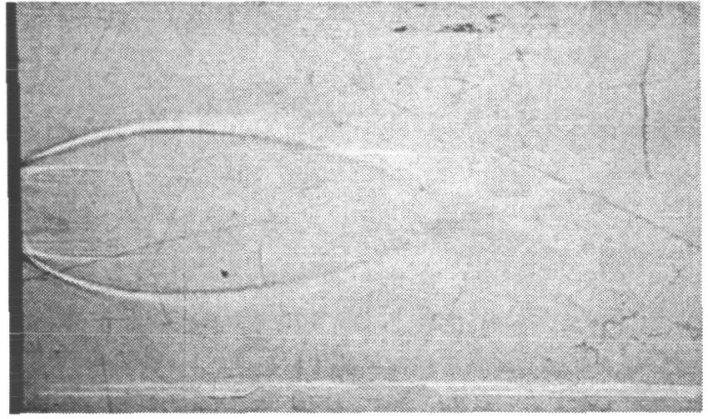

Figure 7. Series of images depicting chamber pressure ramp up for single nozzle strut: (a) Pc $=200$ psi, (b) Pc $=500$ psi, (c) Pc $=600$ psi, and (d) Pc $=700$ psi. 


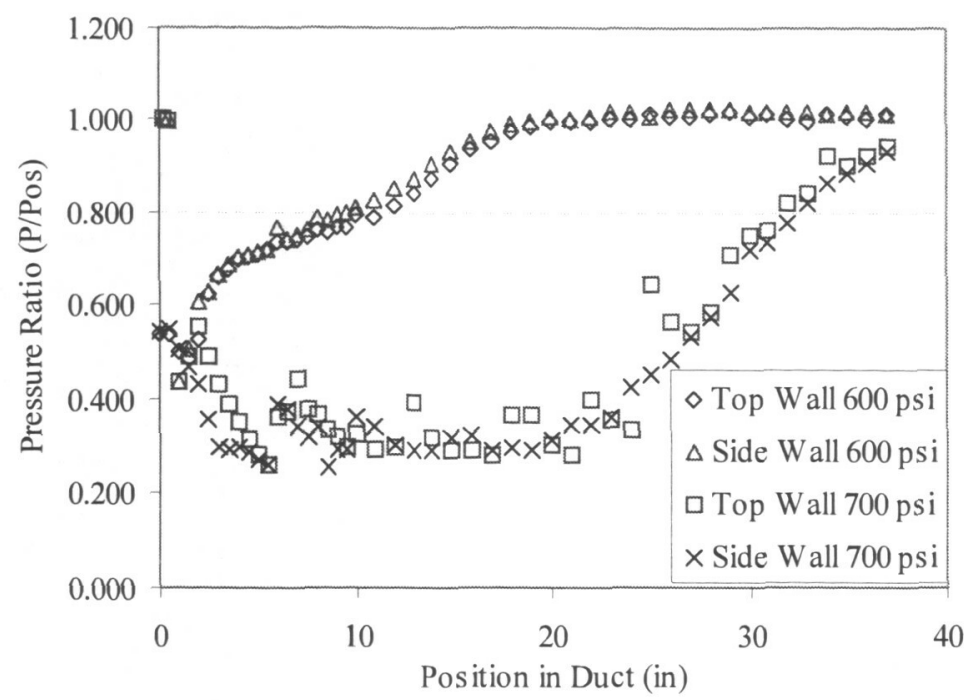

Figure 8. Mixing duct pressure distribution for the single nozzle ejector operated at chamber pressures of $600 \mathrm{psi}$ and $700 \mathrm{psi}$.

Mixing duct pressure profiles were overlaid over the shadowgraph images to see how the showgraphs relate to the measured values. For the low pressure cases (600 psi and less), the overlaid images did not provide much insight as there were no interesting trends in the data. Figure 9 shows a plot of the top wall pressure distribution at a chamber pressure of 700 psi plotted on top of the shadowgraph image. The high pressure trend is clearly visible. In this region of the duct the pressure ratio drops to the value of approximately 0.3 . At approximately 6 inches, there is a sharp discontinuity in the pressure ratio. This discontinuity is believed to be associated with reflected shock structure. If the tails of the barrel shock are extended to the wall, they will reflect at approximately that location.

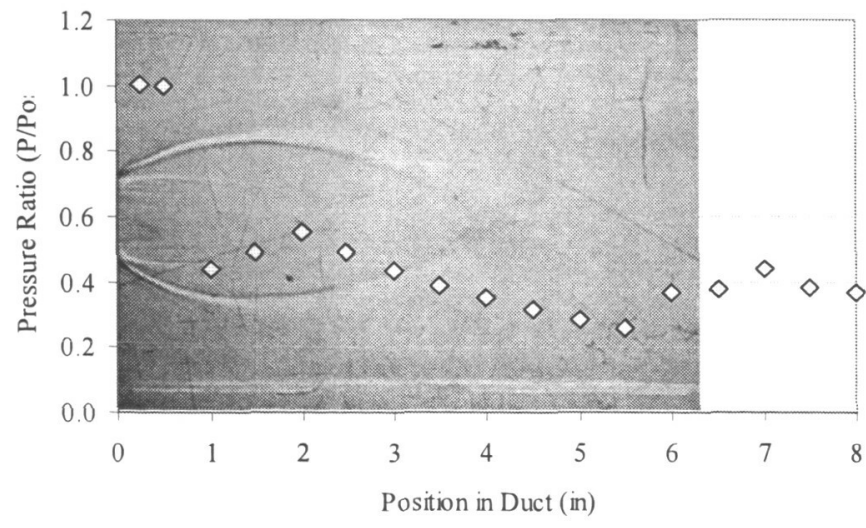

Figure 9. Plot of top wall pressure distribution with shadowgraph image (single nozzle strut $\mathrm{Pc}=700 \mathrm{psi}$ ). 


\section{B. Dual Nozzle Results}

The dual nozzle shadowgraphs revealed a more complex shock structure, especially at high chamber pressures. Figure 10 shows four images of steady state shadowgraphs for the dual nozzle strut at four different chamber pressures. The four images represent the ramp up process for the dual nozzle strut to a chamber pressure of $450 \mathrm{psi}$. Figure 10a shows the steady state image for a chamber pressure of $250 \mathrm{psi}$. Similar to the single nozzle images, barrel shocks emanate from the nozzles, and terminate in Mach disks approximately 1.5 inches downstream. Figure $10 \mathrm{~b}$ shows the shadowgraph image at a steady state chamber pressure of $300 \mathrm{psi}$. The barrel shocks in this image are more stretched out, and still terminate in Mach disks. The barrel shocks for the dual nozzle strut do not appear symmetric, as the inner parts of the shocks are being influence of shocks from the other nozzle. Figure 10c. shows the shadowgraph image at a steady state chamber pressure of $350 \mathrm{psi}$. The barrel shocks are stretched even farther, and the Mach disks have disappeared. For the dual nozzle strut, $350 \mathrm{psi}$ is the chamber pressure around which the transition occurs. Figure 10d shows the shadowgraph image for the steady state chamber pressure of 450 psi. This chamber pressure is above the transition pressure. In the figure, the outer portion of the barrel shocks have extended and intersect approximately 4 inches downstream of the strut. This is approximately the same location where the single nozzle shocks intersected in the 700 psi chamber pressure case. Figure 11 shows the mixing duct pressure distributions for chamber pressures of 300 and 400 psi. These chamber pressures are above and below the transition chamber pressure.

(a)

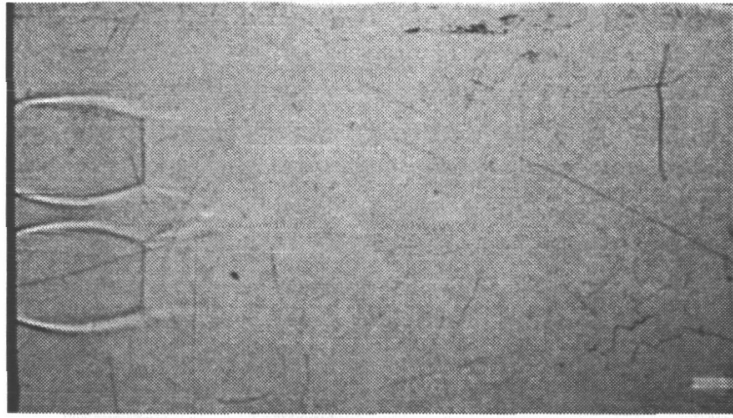

(c)

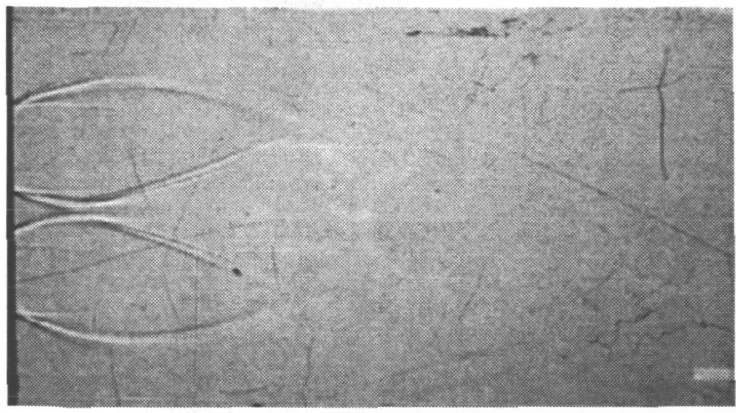

(b)

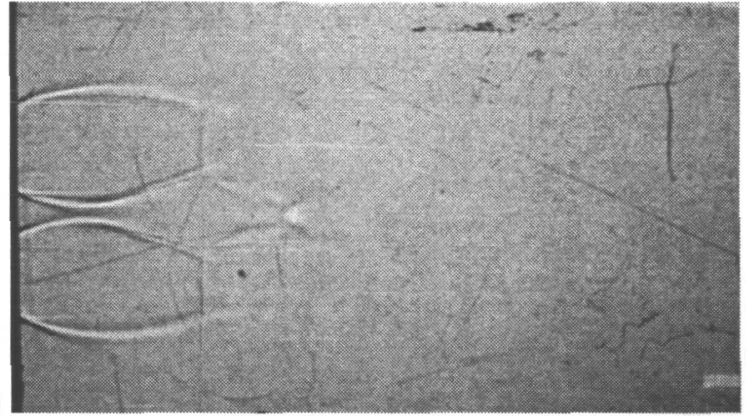

(d)

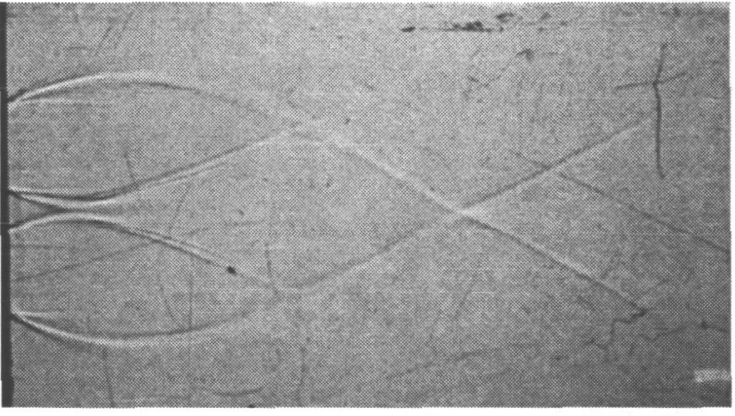

Figure 10. Series of images depicting chamber pressure ramp up for dual nozzle strut: (a) Pc $=250 \mathrm{psi}$, (b) Pc $=$ 300 psi, (c) $\mathrm{Pc}=350 \mathrm{psi}$, and (d) $\mathrm{Pc}=450$ psi. 


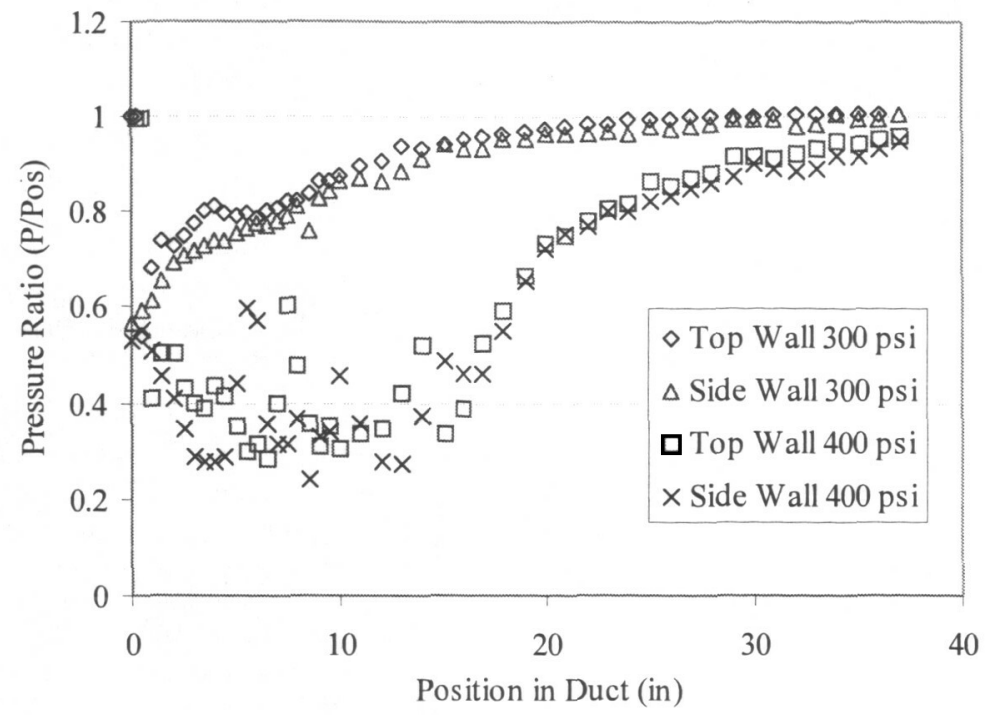

Figure 11. Mixing duct pressure distribution for the dual nozzle ejector operated at chamber pressures of 300 psi and 400 psi.

Mixing duct pressure profiles of the dual nozzle strut were also overlaid over the shadowgraph images to see how the showgraphs related to the measured values. Once again, higher chamber pressures more insightful. Figure 12 shows a plot at 450 psi plotted on top of the shadowgraph image at that pressure. The high pressure trend is again clearly visible. In this region of the duct the pressure ratio is dropping to a constant value of approximately 0.3. At approximately 4 inches and 7 inches, there are discontinuities in the pressure ratio. At each of these locations, there is an increase in the pressure ratio consistent with crossing a shock. If the trailing edges of the barrel shocks are extended to the wall, they will hit the wall at approximately these locations. It is believed that the discontinuities are a result of these shocks. However, extrapolating the length information from the shadowgraph images can be misleading. This point will be discussed more in the conclusions.

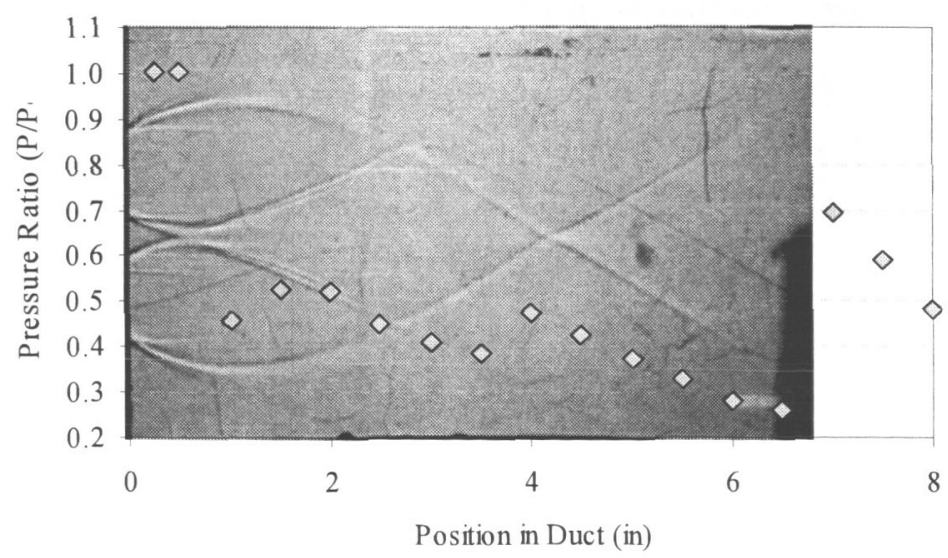

Figure 12. Plot of top wall pressure distribution with shadowgraph image (dual nozzle strut $\mathrm{Pc}=450 \mathrm{psi}$ ). 


\section{Conclusions}

Since the shadowgraphs are of qualitative value, it can be difficult to extract quantitative data that corresponds to the measured parameters. The shadowgraphs provide a two dimensional projection of a three dimensional shock structure, and as a result of this loss of a spatial dimension, information is lost. In other words, the visible image represents a sum of all flow cross sections along the width of the duct, and thus, the pressure readings that are obtained at a specific point may not exactly correspond to the observed shock structure. For this reason, caution must be taken when trying to rely solely on the shadowgraph images for quantitative results.

However, despite the potential difficulties, the shadowgraphs have given useful insight into the nature of this ejector experiment. Given the pressure data that was obtained, discontinuities were apparent and indicated shock interactions within the duct. Use of the shadowgraph images confirmed the presence of shocks at the suspected locations.

Shadowgraphy provides a simple but effective way to obtain additional qualitative information at little to no cost. In this facility, all of the necessary components were either on hand or easily obtained. The principles of this technique allow this setup to be easily modified and can be readily adapted to other facilities desiring to use shadowgraphs. Additionally, shadowgraphy provides a valuable educational tool that augments understanding of complex fluid mechanics.

\section{References}

${ }^{1}$ Settles, G. S., Schlieren and Shadowgraph Techniques Springer, New York, New York, 2001.

${ }^{2 .}$ Murthy, S. N. B. and Curran, E. T., High Speed Flight Propulsion Systems, Progress in Aeronautics and Astronautics Vol. 137, 1991.

${ }^{3}$ Siebenhaar, A. and Bulman, M. J., The Strutjet Engine: The Overlooked Option for Space Launch, in 31st AIAA/ASME/SAE/ASEE Joint Propulsion Conference and Exhibit, San Diego, California, 1995.

${ }^{4}$ Aoki, S., Lee, J., Masuya, G., Kanda, T., and Kudo, K., Aerodynamic Experiment on an Ejector-Jet, Journal of Propulsion and Power 21 (3), 496-503, 2005.

${ }^{5}$. Lineberry, D., Landrum, D. B., Hawk, C., and Smith, N. T., Characterization of Cold Flow NonAxisymmetric Ejectors, in 39th AIAA/ASME/SAE/ASEE Joint Propulsion Conference and Exhibit, Huntsville, Alabama, 2003.

${ }^{6}$ Smith, N. T., An Experimental Investigation of a Non-Axisymmetric Supersonic Cold-Flow Ejector, University of Alabama in Huntsville, 2002.

${ }^{7}$ Muller, S., Bakker, P., Hawk, C., Parkinson, D., and Turner, M., Mixing of Supersonic Jets in a Strutjet Propulsion System, Journal of Propulsion and Power, vol. 17 (No. 5), 1129-1131, 2001.

${ }^{8}$ Landrum, D. B., Thames, M., Parkinson, D., and Gautney, S., Investigation of the Rocket Induced Flow Field in a Rectangular Duct, in 35th AIAA/ASME/SAE/ASEE Joint Propulsion Conference and Exhibit, Los Angeles, California, 1999.

${ }^{9}$. Parkinson, D., Turner, M., and Wagner, D., Mixing of Supersonic Streams, in 35th AIAA/ASME/SAE/ASEE Joint Propulsion Conference and Exhibit, Los Angeles, California, 1999.

${ }^{10 .}$ Lineberry, D. and Landrum, D. B., Effects of Multiple Nozzles on Asymmetric Ejector Performance, in 41th AIAA/ASME/SAE/ASEE Joint Propulsion Conference and Exhibit, Tucson, Arizona, 2005. 